\title{
Endocrine Treatment with 2 Years of Tamoxifen versus 2 Years of Exemestane in Postmenopausal Patients with High-Risk Early Breast Cancer and Persisting Circulating Tumor Cells - First Results of the SUCCESS C Endocrine Treatment Sub-Study
}

\author{
Fabienne Schochter ${ }^{a} \quad$ Brigitte Rack $^{a} \quad$ Marie Tzschaschel $^{a} \quad$ Arkadius Polasik $^{a}$ \\ Ulrich Andergassen $^{b}$ Elisabeth Trapp ${ }^{b}$ Marianna Alunni-Fabbroni ${ }^{b} \quad$ Andreas Schneeweiss $^{c}$
} Volkmar Müller ${ }^{d} \quad K^{2}$ laus Pantel ${ }^{\mathrm{e}}$ Jörg Gade ${ }^{f}$ Ralf Lorenz $^{g}$ Mahdi Rezai ${ }^{\mathrm{h}}$ Hans Tesch $^{\mathrm{i}}$ Ulrike Soeling $^{j}$ Tanja Fehm $^{k}$ Sven Mahner ${ }^{b}$ Christian Schindlbeck' Werner Lichtenegger ${ }^{m}$ Matthias W. Beckmann ${ }^{\mathrm{n}}$ Peter A. Fasching ${ }^{\mathrm{n}}$ Wolfgang Janni ${ }^{\mathrm{a}}$ Thomas W.P. Friedl ${ }^{\mathrm{a}}$ on behalf of the SUCCESS Study Group

\footnotetext{
a Universitätsfrauenklinik UIm, Ulm, Germany;

${ }^{b}$ Klinik und Poliklinik für Frauenheilkunde und Geburtshilfe, Klinikum der Ludwig-Maximilians-Universität München, Munich, Germany;

${ }^{\mathrm{c}}$ Nationales Zentrum für Tumorerkrankungen, Universitätsklinikum Heidelberg, Heidelberg, Germany;

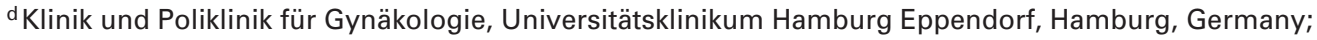

e Institut für Tumorbiologie, Universitätsklinikum Hamburg Eppendorf, Hamburg, Germany;

${ }^{f}$ Klinik für Gynäkologie, Diakoniekrankenhaus Friederikenstift, Hannover, Germany;

g Gemeinschaftspraxis Dr. Lorenz, Hecker und Wesche, Braunschweig, Germany;

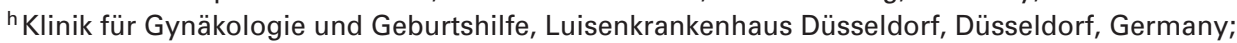

' Onkologische Gemeinschaftspraxis, Frankfurt/M., Germany;

j Gemeinschaftspraxis Siehl und Soeling, Kassel, Germany;

k Klinik für Frauenheilkunde und Geburtshilfe, Klinikum der Heinrich-Heine-Universität Düsseldorf, Düsseldorf, Germany;

' Frauenklinik, Klinikum Traunstein, Traunstein, Germany;

m Charité Universitätsklinikum, Campus Virchow, Berlin, Germany;

${ }^{n}$ Frauenklinik, Friedrich-Alexander Universität Erlangen-Nürnberg, Universitätsklinikum Erlangen, Erlangen, Germany
}

\section{Keywords}

Early breast cancer - Endocrine therapy · Exemestane .

Tamoxifen - Circulating tumor cells - CTC clearance rate . Safety $\cdot$ Toxicity

\section{Summary}

Background: Optimal choice and sequence of endocrine treatment following adjuvant chemotherapy in postmenopausal early breast cancer patients are still under discussion and treatment stratification factors are missing. Patients and Methods: Postmenopausal women with HER2negative, hormone receptor-positive tumors and persisting circulating tumor cells (CTCs; assessed using the FDA-approved CellSearch ${ }^{\circledR}$ System, Janssen Diagnostics, LLC) after chemotherapy were randomized to 2 years of tamoxifen followed by 3 years of exemestane (tamoxifen- exemestane group, $n=54$ ) or 5 years of exemestane (exemestane-only group, $\mathrm{n}=54$ ). CTCs were again assessed after the first 2 years of endocrine treatment. In addition, safety data were compared between the 2 groups. Results: The 2 groups were well-balanced with regard to baseline characteristics. The CTC clearance rate after 2 years was $89 \%$ in the exemestane-only group and $97 \%$ in the tamoxifen-exemestane group (exact Fisher test, $p=0.36)$. The safety profile showed good tolerability with few grade 3 or 4 adverse events in both groups. Conclusion: The similar CTC clearance rate after 2 years of endocrine therapy with exemestane or tamoxifen, and the safety profiles obtained may indicate comparable efficacy and tolerability of both endocrine treatment regimens. However, these results have to be confirmed by final survival and safety analysis.

(c) 2018 S. Karger GmbH, Freiburg

\section{KARGER}

(C) 2018 S. Karger GmbH, Freiburg 


\section{Introduction}

For many years, treatment with the selective estrogen receptor (ER) modulator tamoxifen was a cornerstone of adjuvant therapy in postmenopausal patients with hormone receptor (HR)-positive breast cancer (BC). However, the development of aromatase inhibitors (AIs) that prevent the synthesis of estrogen from androgens provided clinicians with a powerful alternative endocrine treatment option. There are 2 types of third generation AIs currently available for treatment of HR-positive BC. Type I AIs (exemestane) are steroidal permanent inhibitors, and type II AIs (anastrozole, letrozole) are non-steroidal reversible competitive inhibitors. Several trials have compared tamoxifen with AIs in the adjuvant treatment of HR-positive BC. A recent systematic review and meta-analysis revealed significantly improved disease-free survival (DFS) and overall survival (OS) for 5 years of AI treatment compared to 5 years of tamoxifen with high and moderate quality of evidence, respectively, albeit with rather small absolute differences [1]. In addition, the meta-analysis revealed a significant but small DFS benefit for the sequential tamoxifen-AI regimen compared to tamoxifen monotherapy, but no significant difference in OS (though the difference approached significance) [1]. Altogether, the evidence so far indicates that AIs (either as monotherapy or following 2 years of tamoxifen) are superior to 5 years of tamoxifen monotherapy as endocrine treatment of postmenopausal BC patients, even if the gain in DFS is small and clear evidence regarding an OS benefit is still lacking [1-3]. However, it is still unknown whether a sequential regimen (tamoxifen followed by AI or vice versa) or an $\mathrm{AI}$ monotherapy is to be preferred, as there is no convincing evidence suggesting significant differences with respect to both DFS and OS between these 2 treatment regimen [1]. Metaanalyses of endocrine treatment-related adverse events showed that AIs were associated with a higher incidence of bone fractures, while tamoxifen was associated with a higher risk of endometrial cancer and venous thrombosis; in addition, sequential therapy with tamoxifen followed by AI was associated with a lower risk of developing cardiovascular disease compared to AI monotherapy $[1,4]$. Thus, the decision between sequential treatment and AI monotherapy may be based on the differences in the toxicity profile and preexisting risk factors.

Dissemination of circulating tumor cells (CTCs) from the primary tumor into the bloodstream is considered a risk factor and a critical step in tumorigenesis as CTCs are regarded a precursor of metastases by initiating tumor growth in distant organs $[5,6]$. Several studies have revealed the independent prognostic value of CTCs assessed before the start of adjuvant chemotherapy in early $\mathrm{BC}$ (EBC) [7-10]. The independent prognostic relevance was also shown for the presence of CTCs after adjuvant chemotherapy $[7,11]$. Recently, it was also demonstrated that persistence of CTCs assessed during follow-up care 2 years after adjuvant chemotherapy is associated with poor outcome in high-risk EBC patients [12].

The aim of this study is to present data regarding the comparison between a sequential endocrine treatment regimen ( 2 years ta- moxifen followed by 3 years exemestane) and an AI monotherapy (5 years exemestane) in postmenopausal women with HER2-negative EBC and evidence of CTCs in the peripheral blood at the time of the last chemotherapy cycle (indicating increased risk of recurrence). This analysis represents the first results of the endocrine treatment sub-study of the randomized phase III German SUCCESS C trial, which compares anthracycline-containing and anthracycline-free chemotherapy in women with HER2-negative EBC. The endocrine treatment sub-study of SUCCESS C represents oneof the first clinical trials in which the presence of CTCs as a validated risk marker was used as an inclusion criterion for a subsequent therapy randomization. We evaluated efficacy assessed based on the presence of CTCs and CTC clearance rate 2 years after the start of endocrine treatment as an early prognostic surrogate marker for clinical outcome. Thus, this first analysis evaluates efficacy after 2 years of tamoxifen versus 2 years of exemestane. In addition, we present the first data on safety in terms of the occurrence of adverse events during endocrine treatment.

\section{Patients and Methods}

Study Design

SUCCESS C (NCT00847444) is a randomized multicenter open-label phase III clinical trial for women with histologically confirmed HER2-negative, highrisk primary $\mathrm{BC}$ with an indication for adjuvant chemotherapy (either positive lymph nodes or high-risk node-negative BC, defined as tumor size larger than $2 \mathrm{~cm}$, histopathological grade 3 , age $\leq 35$ years or HR-negative primary tumor).

The primary objectives of this study were to assess the efficacy of an anthracycline-free adjuvant chemotherapy regimen in HER2-negative BC and to evaluate the effect of a lifestyle intervention program on DFS of EBC patients. A blood sample was collected immediately before the last chemotherapy application for CTC assessment (see below) from all patients with HR-positive tumors who provided written informed consent for blood sampling as part of the translational research program of SUCCESS C. Determination of bone density before the start of therapy was not performed within the SUCCESS C study. According to the study protocol, bisphosphonates were not recommended, but the administration of bisphosphonates was admitted.

Postmenopausal HR-positive patients with evidence of CTCs in the peripheral blood at the time of the last chemotherapy cycle were eligible for the endocrine treatment sub-study. Given that they provided written informed consent for this sub-study, these patients were randomized to 5 years of treatment with the AI exemestane ( $25 \mathrm{mg}$ p.o. per day; exemestane-only group) versus 2 years of tamoxifen ( $20 \mathrm{mg}$ p.o. per day) followed by 3 years of exemestane (tamoxifen-exemestane group). Postmenopausal HR-positive patients without CTCs (and patients who did not provide written informed consent for the endocrine treatment sub-study) received exemestane ( $25 \mathrm{mg}$ p.o. per day) for 5 years. Patients with HR-positive primary tumor under the age of 40 and/or with premenopausal hormone levels (luteinizing hormone $(\mathrm{LH})<20 \mathrm{mlU} / \mathrm{ml}$, folliclestimulating hormone $(\mathrm{FSH})<20 \mathrm{mlU} / \mathrm{ml}$ and estradiol $(\mathrm{E} 2)>20 \mathrm{pg} / \mathrm{ml}$ ) received goserelin (3.6 mg subcutaneously, every 4 weeks) for 2 years and tamoxifen (20 mg p.o. per day) for 5 years. The endocrine therapy schedule in SUCCESS C including the endocrine treatment sub-study is shown in Supplemental Fig. 1 (www.karger.com/?DOI=485566); the complete study design and more detailed information regarding trial concept, inclusion and exclusion criteria, life-style intervention and translational research program is provided elsewhere [13].

Patients

Overall, 3,642 patients were recruited to the SUCCESS C trial. Data on CTC prevalence at the date of last application of chemotherapy were available from 

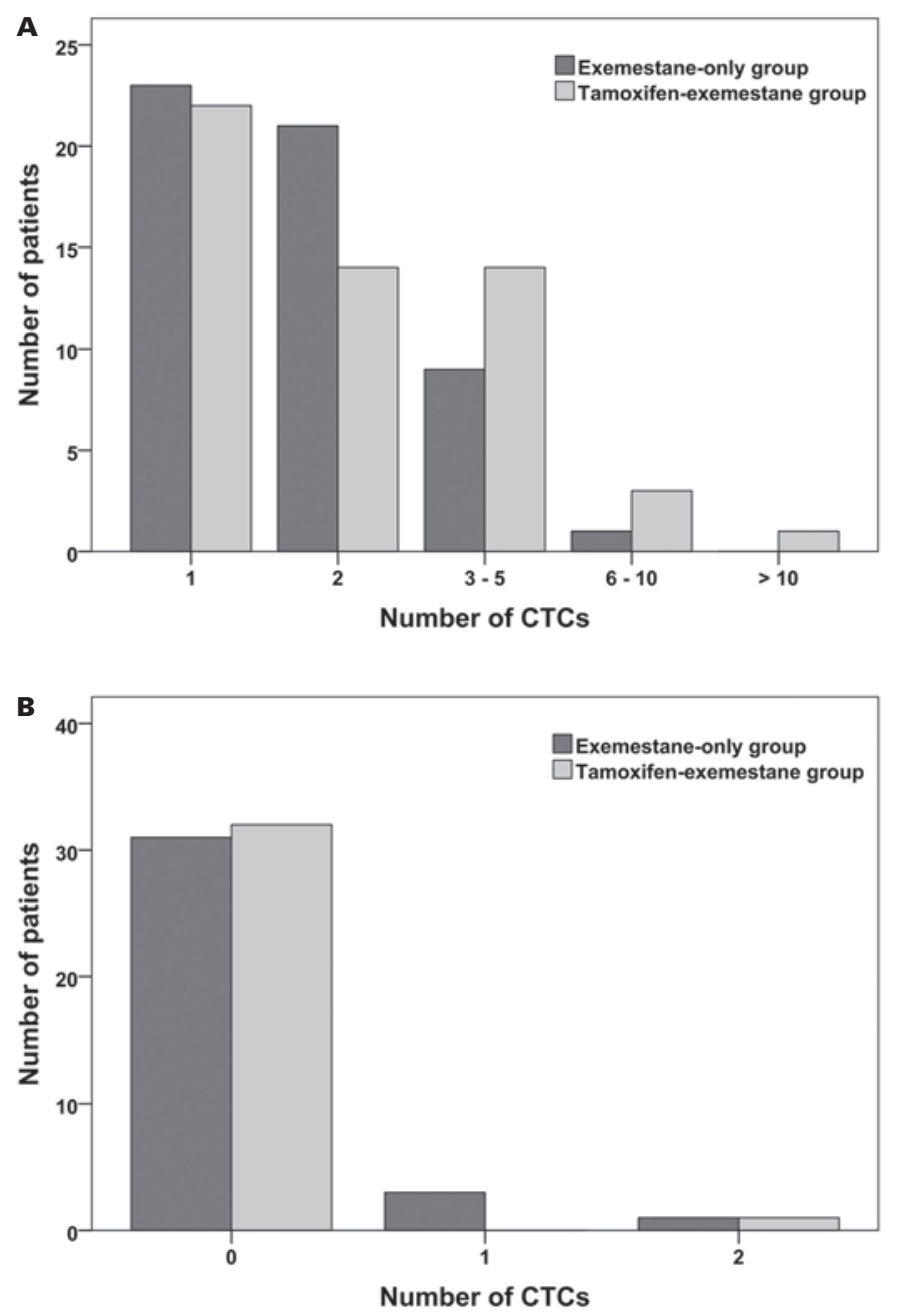

Fig. 1. Frequency distribution of the number of circulating tumor cells (CTCs) detected according to randomization arm in postmenopausal patients with HER2-negative, hormone receptor-positive early breast cancer (EBC) patients randomized in the endocrine treatment sub-study of the SUCCESS $C$ trial. A At the time of the last chemotherapy cycle $(\mathrm{n}=108)$; B 2 years after adjuvant chemotherapy $(\mathrm{n}=68)$.
1,766 patients, and in 221 (12.5\%) patients at least 1 CTC was detected. Out of 136 CTC-positive patients with a postmenopausal status, 108 patients provided written informed consent and could be randomized for the endocrine treatment sub-study (see Supplemental Fig. 2 (www.karger.com/?DOI=485566)). The study was conducted in accordance with the Declaration of Helsinki and Good Clinical Practice Guidelines, and was approved by all responsible ethic committees.

\section{Tumor Characteristics and Safety Data}

Tumor stages at primary diagnosis were classified according to the revised American Joint Committee on Cancer TNM classification system [14]. Histological grading was classified according to the Elston-Ellis modification of the Scarff-Bloom-Richardson grading system [15]. Tumors were defined as HRpositive if the percentage of cells with immunohistochemical nuclear staining for estrogen and/or progesterone receptor was $10 \%$ or higher. HER 2 negativity of the primary tumor was defined by negative immunohistochemistry (IHC) value of $0-1+$ and/or negative fluorescent in situ hybridization.

Safety data were assessed according to the Common Terminology Criteria for Adverse Events of the National Cancer Institute, CTCAE Version 3.0. For the safety analyses reported here, all adverse and serious adverse events were included that occurred after randomization for the endocrine treatment substudy, which took place after the results of CTC assessment at the time of the last chemotherapy cycle were available (i.e. shortly before the start of endocrine treatment).

\section{Detection of CTCs}

As part of a SUCCESS C translational research sub-project, peripheral blood samples for CTC determination were collected from patients with HRpositive tumors at the end of the systemic treatment (immediately before the last chemotherapy infusion or, in patients with early discontinuation of chemotherapy, 3 weeks after the last chemotherapy application). CTC analyses were performed using the FDA-approved CellSearch System ${ }^{\circledR}$ (Janssen Diagnostics, Raritan, USA) as described in detail elsewhere [16]. Samples with at least 1 detected CTC were considered CTC positive.

\section{Data Analysis}

The analysis reported here is a non-preplanned interim analysis presenting first results of the endocrine treatment sub-study of the SUCCESS C trial. Data export for this analysis from the central data management database was conducted in April 2016. Data analysis is based on the intention-to-treat principle.
Table 1. Number of patients (\%) affected by, and absolute frequencies of, the most common adverse events (Common Terminology Criteria for Adverse Events CTCAE v3.0) observed in the endocrine treatment sub-study of SUCCESSS C according to grade and randomization arm

\begin{tabular}{|c|c|c|c|c|}
\hline \multirow[t]{2}{*}{$\begin{array}{l}\text { Adverse event term } \\
\text { (CTCAE v3.0) }\end{array}$} & \multicolumn{2}{|c|}{ Exemestane-only group $(\mathrm{n}=54)$} & \multicolumn{2}{|c|}{$\begin{array}{l}\text { Tamoxifen-exemestane group } \\
(\mathrm{n}=54)\end{array}$} \\
\hline & Grade 1 or 2 & Grade 3 or 4 & Grade 1 or 2 & Grade 3 or 4 \\
\hline Leukopenia & $6(11.1), 13$ & & $6(11.1), 6$ & \\
\hline Hypertension & $3(5.6), 3$ & & $7(13.0), 8$ & \\
\hline Fatigue & $5(9.3), 5$ & & $6(11.1), 11$ & $1(1.9), 1$ \\
\hline Insomnia & $5(9.3), 6$ & & $5(9.3), 6$ & $1(1.9), 1$ \\
\hline Alopecia & $5(9.3), 6$ & & $4(7.4), 5$ & \\
\hline Hot flushes & $6(11.1), 7$ & & $6(11.1), 10$ & $1(1.9), 1$ \\
\hline Hypercholesteremia & $10(18.5), 13$ & & $9(16.7), 14$ & \\
\hline Creatinine & $1(1.9), 9$ & & $2(3.7), 3$ & \\
\hline$\gamma$-Glutamyl transpeptidase & 7 (13.0), 8 & & $7(13.0), 10$ & \\
\hline Hyperglycemia & $9(16.7), 12$ & & $11(20.4), 15$ & \\
\hline Hypertriglyceridemia & $5(9.3), 7$ & & $6(11.1), 10$ & \\
\hline Mood alteration: depression & $6(11.1), 7$ & & $3(5.6), 3$ & \\
\hline Neuropathy: sensory & $5(9.3), 5$ & $1(1.9), 1$ & $8(14.8), 9$ & \\
\hline Bone pain & $8(14.8), 9$ & $1(1.9), 1$ & $9(16.7), 14$ & $2(3.7), 2$ \\
\hline Joint pain & $12(22.2), 16$ & & $7(13.0), 10$ & \\
\hline
\end{tabular}


All categorical variables are described in terms of absolute and relative frequencies, while the non-normally distributed continuous variables age and body mass index (BMI) as well as number of CTCs detected are described by reporting medians and ranges.

Associations between the presence of CTCs and patient as well as tumor characteristics were analyzed using the Mann-Whitney U-test for age and BMI, and the chi-square test for all categorical variables. Comparisons of adverse event frequencies between the 2 randomization arms (proportion of patients affected) were performed with chi-square tests (or Fisher's exact test in case of expected frequencies of 5 or less in at least 1 cell of the $2 \times 2$ contingency tables). All statistical tests were 2 -sided, and p values of less than 0.05 were considered significant (no adjustments of significance levels for multiple testing). Statistical analyses were performed using IBM SPSS Statistics, Version $21.0 \mathrm{~s}$ (IBM Corp., Armonk, New York, USA).

\section{Results}

\section{CTC Prevalence and CTC Clearance Rates}

Patient and tumor characteristics were well balanced between the 2 randomization arms of the endocrine treatment sub-study (see Supplemental Table 1 (www.karger.com/?DOI=485566)). CTC prevalence at the end of the systemic treatment was low even in the CTC-positive patient cohort eligible for this sub-study (median 2 CTCs, range 1-12 CTCs), and there was no significant difference between the 2 randomization arms with regard to the number of CTCs detected (Mann-Whitney U-test, $\mathrm{p}=0.30$; fig. 1A). A CTC count 2 years after adjuvant chemotherapy was obtained for 68 patients ( 35 patients in the exemestane-only group, 33 patients in the tamoxifen-exemestane group). In the exemestane-only group, no CTCs were detected in 31 patients, while 1 CTC was detected in 3 patients and 2 CTCs were detected in 1 patient; in the tamoxifenexemestane group, no CTCs were found in 32 patients and 1 patient had 2 CTCs (fig. 1B). Thus, the CTC clearance rates after 2 years were $89 \%$ in the exemestane-only group and $97 \%$ in the tamoxifen-exemestane group, which was not significantly different (exact Fisher test, $\mathrm{p}=0.36$ ).

\section{Safety}

Overall, 585 adverse events were recorded in patients after they were randomized for the endocrine treatment sub-study. 283 adverse events occurred in patients of the exemestane-only group ( $\mathrm{n}=54$ patients) and 302 adverse events occurred in patients of the tamoxifen-exemestane group ( $\mathrm{n}=54$ patients). In the exemestaneonly group, there were 207 (73.1\%) grade 1, 63 (22.3\%) grade 2, 11 (3.9\%) grade 3 and $2(0.7 \%)$ grade 4 adverse events. Similarly, in the tamoxifen-exemestane group 217 (71.9\%) grade 1, 68 (22.5\%) grade 2, and 17 (5.6\%) grade 3 adverse events occurred; no grade 4 adverse event was observed. Supplemental Table 2 ( $w w w . k a r g e r$. $c o m / ? D O I=485566)$ shows numbers and percentages of patients affected by adverse events according to category (CTCAE v3.0) as well as their absolute frequencies, grade and randomization arm. There were no significant differences between the 2 randomization arms with regard to the proportion of patients affected for any of the adverse event categories (all $\mathrm{p}>0.05$ ). The most common adverse events with an overall absolute frequency of 10 or higher are listed in table 1; again, there were no significant differences between the 2 groups with regard to the proportion of patients affected by these adverse events (all $\mathrm{p}>0.15$ ).

In total, 52 adverse events (only grade 1 or 2 ) were regarded as being very likely associated with endocrine treatment ( 23 in the exemestane-only group, 29 in the tamoxifen-exemestane group). A likely association with endocrine treatment was stated for 55 adverse events (30 in the exemestane-only group, 25 in the tamoxifen-exemestane group), all of which were grade 1 or 2 with the exception of one grade 3 bone pain event in the tamoxifen-exemestane group. For 154 adverse events (74 in the exemestane-only group, 80 in the tamoxifen-exemestane group), association with endocrine treatment was rated as being possible. With the exception of 1 grade 3 bone pain event in the exemestane-only group, and 1 grade 3 fatigue and 1 grade 3 dizziness event in the tamoxifen-exemestane group, all these adverse events were grade 1 or 2 only. The remaining 324 adverse events (including 24 grade 3 and 2 grade 4 events) were categorized as not likely being associated with, or not being associated with, endocrine treatment.

\section{Discussion}

Our preliminary efficacy analysis of the endocrine treatment sub-study of the SUCCESS C trial revealed no difference in CTC clearance rate between 2 years of endocrine treatment with either tamoxifen or exemestane in postmenopausal women with EBC who were at increased risk of recurrence (as assessed by the presence of CTCs at the time of the last chemotherapy cycle). The preliminary safety analysis showed a comparable safety profile and good tolerability with only very few grade 3 or 4 adverse events in both the sequential endocrine regimen with 2 years of tamoxifen followed by 3 years of exemestane (tamoxifen-exemestane group) and the 5-year endocrine monotherapy with exemestane (exemestane-only group).

A recent meta-analysis combining data from the 2 large randomized trials comparing 5-year endocrine treatments with tamoxifen or AI (BIG-01-98, ATAC) revealed significantly improved DFS and OS for patients receiving AI monotherapy [1]. Our analysis evaluating CTC prevalence after 2 years of tamoxifen versus 2 years of exemestane treatment did not show a significant difference in CTC clearance rate between the 2 treatments. Since presence of CTCs 2 years after adjuvant chemotherapy is an independent prognostic factor for poor outcome in EBC patients [12], our results may indicate equal efficacy of both treatment regimen in a high-risk collective of HER2-negative BC patients, at least when assessed 2 years after start of endocrine treatment. This result is in contrast to subgroup analyses of the BIG-01-98 trial, showing that the benefit for AI was most pronounced in high-risk (node-positive) patients, while the hazard ratios were not significant for nodenegative patients [17].

Two large trials investigated efficacy and safety of a sequential tamoxifen followed by AI regimen compared to 5 years of AI monotherapy - similar to the design of the endocrine treatment sub- 
study of the SUCCESS C trial reported here - and thus provided suitable data for comparison. The BIG 01-98/IBCSG 18-98 trial (NCT00004205) compared 5 years of tamoxifen or letrozole monotherapy, or sequential treatment with 2 years of one of these drugs followed by 3 years of the other in postmenopausal women with HR-positive BC. The analysis regarding the comparison of 5 years of letrozole monotherapy with each of the 2 sequential regimen (tamoxifen followed by letrozole or vice versa) revealed no statistically significant differences with regard to DFS, OS, distant recurrence-free interval, or BC-free interval [17]. The TEAM (Tamoxifen Exemestane Adjuvant Multinational) trial (NCT00036270) compared 5 years of exemestane monotherapy and a sequential treatment regimen with 2.5-3.0 years of tamoxifen followed by exemestane for a total of 5 years in postmenopausal HR-positive BC. Similar to the results of BIG 01-98, no significant differences between the 2 arms with respect to DFS or OS were found [18]. Taken together, the results of both BIG 01-98 and TEAM suggest that a sequential 5-year endocrine treatment regimen with tamoxifen followed by an AI may be equally effective as 5-year AI monotherapy in postmenopausal primary BC patients.

The safety analysis of the BIG 01-98 trial and the TEAM trial provided comparable results regarding toxicity and tolerability of sequential treatments with tamoxifen followed by AI compared to AI monotherapy. While the sequential treatments with tamoxifen were associated with higher rates of thromboembolic events, gynecological symptoms (such as vaginal bleeding) and endometrial abnormalities (including endometrial cancer), AI monotherapy led to higher rates of musculoskeletal adverse events (in particular bone fractures), hypercholesterolemia or hyperlipidemia, and hypertension $[18,19]$. Similar results were obtained in a meta-analysis evaluating toxicity of adjuvant endocrine therapy in postmenopausal BC patients [4]. Our analysis indicated good tolerability with only very few grade 3 or 4 adverse events in both the tamoxifen-exemestane group and the exemestane-only group.

Several recent studies have confirmed the suitability of CTCs as assessed using the FDA-cleared CellSearch ${ }^{\circledR}$ System to serve as prognostic and/or predictive marker in EBC. The strong independent prognostic value of CTCs in the peripheral blood of BC patients before the start of adjuvant chemotherapy has been demonstrated in a recent large pooled analysis of individual data from 3,173 patients, showing that the presence of at least 1 CTC was significantly associated with decreased OS, BC-specific survival, DFS, and distant DFS [10]. Presence of CTCs after adjuvant chemotherapy is also associated with poor outcome $[7,11]$, and eligibility for the endocrine treatment sub-study was based on the presence of CTCs at the time of the last chemotherapy cycle as indicator of increased risk of recurrence. While the individual CTC counts after adjuvant chemotherapy as found in our study appear to be rather low, they are similar to the CTC counts after chemotherapy in the 2 studies that reported a significant association between the presence of at least 1 CTC after adjuvant chemotherapy and decreased DFS and/or OS $[7,11]$. Thus, we are confident that the positive CTC count after adjuvant chemotherapy used as a marker for increased risk of recurrence in our study indeed represents a mean- ingful prognostic marker. Importantly, recent data provide strong evidence that the presence of CTCs 2 years after adjuvant chemotherapy is an independent prognostic factor for poor OS and DFS [12]. These data indicate that CTCs assessed 2 years after adjuvant chemotherapy may be used as easily accessible (by liquid biopsy) surveillance marker during routine long-term follow up of BC patients to guide risk-adapted tailored follow-up care.

While the SUCCESS C endocrine treatment sub-study is novel because it is one of the first trials in which inclusion and randomization to different therapies is contingent on the presence of CTCs as established risk factor for an increased risk of recurrence, there are also some limitations with regard to our first analysis of the endocrine treatment sub-study. Most obviously, the analysis is limited by the small number of patients that could be randomized for this study. In addition, it has to be noted that CTC prevalence after 2 years only serves as an early available surrogate marker for outcome. Furthermore, as CTC prevalence was determined 2 years after start of the endocrine treatment, this efficacy analysis compares 2 years of tamoxifen and 2 years of exemestane (and thus does not represent a comparison between a 5-year sequential endocrine treatment with tamoxifen followed by an AI and a 5-year AI monotherapy as was the primary objective of the endocrine treatment substudy). Two years of endocrine treatment is rather short and represents not the standard of care in a high-risk population of BC patients such as those included in this endocrine treatment sub-study. Thus, both efficacy and safety data reported here have to be regarded as preliminary and as such have to be interpreted with care.

In conclusion, we could not find a significant difference between endocrine treatment with tamoxifen and exemestane with regard to CTC clearance rate 2 years after start of endocrine treatment in postmenopausal HR-positive EBC patients with CTCs at the time of the last adjuvant chemotherapy cycle (indicating an increased risk of recurrence). The safety profile obtained so far indicated good tolerability in both the tamoxifen-exemestane group and the exemestane-only group. Our preliminary results thus suggest comparable efficacy and tolerability of both endocrine treatment regimen. However, final analyses of the endocrine treatment sub-study with regard to both survival and safety based on longer follow up data have to be awaited to be able to draw firm conclusions regarding the efficacy and tolerability of a 5-year sequential endocrine treatment with tamoxifen followed by an $\mathrm{AI}$ as compared to a 5-year AI monotherapy in postmenopausal EBC patients with an increased risk of recurrence.

\section{Online Supplemental Material}

Supplemental Table 1: Patient and tumor characteristics of postmenopausal patients with HER2-negative, hormone receptor-positive early breast cancer who had at least 1 CTC in the peripheral blood at the time of the last chemotherapy cycle and were randomized for the endocrine treatment substudy of the SUCCESS C clinical trial.

Supplemental Table 2 Number of patients (\%) affected by and absolute frequencies of adverse events observed in the endocrine treatment substudy of SUCCESSS C according to category (Common Terminology Criteria for Adverse Events (CTCAE) v3.0), grade and randomization arm. 
Supplemental Fig. 1. Endocrine therapy schedule and design of the endocrine treatment sub-study of the SUCCESS C clinical trial.

Supplemental Fig. 2. Flow chart of patient selection. CTC: Circulating tumor cell; HR: hormone receptor.

To access the online supplemental material, please refer to ( $w w w . k a r g e r$. $\mathrm{com} /$ ?DOI=485566).

\section{Acknowledgements}

The SUCCESS C study was funded by grants from Chugai, Glaxo Smith Kline, Pfizer, Sanofi-Aventis and Veridex (now Janssen Diagnostics). We thank all participating patients and study centers of the SUCCESS C study.

\section{Disclosure Statement}

B. Rack received financial research support from Sanofi-Aventis, Chugai, Pfizer, GlaxoSmithKline and Veridex (Janssen Diagnostics). H. Tesch has a consulting role for and received fees from Roche, Amgen, Pfizer and Novartis. S. Mahner received research support, honoraria and/or travel expenses from AstraZeneca, Bayer, Boehringer Ingelheim, Clovis, GlaxoSmithKline, Jenapharm, Janssen-Cilag, Medac, MSD, PharmaMar, Roche, Sensor Kinesis, Teva, and Tesaro. P.A. Fasching received research funding from Novartis, honoraria from Novartis, Roche, Pfizer and Celgene and has a consulting role for Novartis, Roche, Pfizer and Celgene. W. Janni received research funding and honoraria from Sanofi-Aventis, Pfizer, Novartis, AstraZeneca, and Lilly. F. Schochter, M. Tzschaschel, A. Polasik, U. Andergassen, E. Trapp, M. AlunniFabbroni, A. Schneeweiss, V. Müller, K. Pantel, J. Gade, R. Lorenz, M. Rezai, U. Söling, T. Fehm, C. Schindlbeck, W. Lichtenegger, M.W. Beckmann and T.W.P. Friedl have no conflicts of interest.

\section{References}

1 Ryden L, Arnlind M, Vitols S, et al.: Aromatase inhibitors alone or sequentially combined with tamoxifen in postmenopausal early breast cancer compared with tamoxifen or placebo - Meta-analyses on efficacy and adverse events based on randomized clinical trials. Breast 2016;26:106-114

2 Janni W, Hepp P: Adjuvant aromatase inhibitor therapy: Outcomes and safety. Cancer Treat Rev 2010;36: 249-261.

3 Freedman OC, Fletcher GG, Gandhi S, et al.: Adjuvan endocrine therapy for early breast cancer: A systematic review of the evidence for the 2014 Cancer Care Ontario systemic therapy guideline. Curr Oncol 2015; 22:S95-S113.

4 Amir E, Seruga B, Niraula S, et al.: Toxicity of adjuvant endocrine therapy in postmenopausal breast cancer patients: A systematic review and meta-analysis. J Natl Cancer Inst 2011;103:1299-1309.

5 Banys-Paluchowski M, Krawczyk N, Meier-Stiegen F, Fehm T: Circulating tumor cells in breast cancer-current status and perspectives. Crit Rev Oncol Hematol 2016;97:22-29.

6 Bednarz-Knoll N, Alix-Panabieres C, Pantel K: Clinical relevance and biology of circulating tumor cells. Breast Cancer Res 2011;13:228.
7 Rack B, Schindlbeck C, Jückstock J, et al.: Circulating tumor cells predict survival in early average-to-high risk breast cancer patients. J Natl Cancer Inst 2014; 106:pii:dju066.

8 Pierga JY, Bidard FC, Mathiot C, et al.: Circulating tumor cell detection predicts early metastatic relapse after neoadjuvant chemotherapy in large operable and locally advanced breast cancer in a phase II randomized trial. Clin Cancer Res 2008;14:7004-7010.

9 Lucci A, Hall CS, Lodhi AK, et al.: Circulating tumour cells in non-metastatic breast cancer: A prospective study. Lancet Oncol 2012;13:688-695.

10 Janni WJ, Rack B, Terstappen LW, et al.: Pooled analysis of the prognostic relevance of circulating tumor cells in primary breast cancer. Clin Cancer Res 2016; 22:2583-2593.

11 van Dalum G, van der Stam GJ, Tibbe AGJ, et al.: Circulating tumor cells before and during follow-up after breast cancer surgery. Int J Oncol 2015;46:407-413.

12 Janni W, Rack B, Fasching P, et al.: Persistence of circulating tumor cells in high risk early breast cancer patients during follow-up care suggests poor prognosis - Results from the adjuvant SUCCESS A trial. Cancer Res 2016;76:S2-3

13 Rack B, Andergassen U, Neugebauer J, et al.: The German SUCCESS C Study - The first European lifestyle study on breast cancer. Breast Care (Basel) 2010;5: 395-400.
14 Edge S, Byrd DR, Compton CC, et al. (eds): AJCC Cancer Staging Manual, 7th ed. New York, Springer, 2010.

15 Elston CW, Ellis IO: Pathological prognostic factors in breast cancer. I. The value of histological grade in breast cancer: Experience from a large study with longterm follow-up. Histopathology 1991;19:403-410.

16 Riethdorf S, Fritsche H, Müller V, et al.: Detection of circulating tumor cells in peripheral blood of patients with metastatic breast cancer: A validation study of the CellSearch system. Clin Cancer Res 2007;13:920-928.

17 Regan MM, Neven P, Giobbie-Hurder A, et al.: Assessment of letrozole and tamoxifen alone and in sequence for postmenopausal women with steroid hormone receptor-positive breast cancer: The BIG 1-98 randomised clinical trial at 8.1 years median follow-up. Lancet Oncol 2011;12:1101-1108.

18 Van De Velde CJ, Rea D, Seynaeve C, et al.: Adjuvant tamoxifen and exemestane in early breast cancer (TEAM): A randomised phase 3 trial. Lancet 2011;377: 321-331.

19 BIG 1-98 Collaborative Group, Mouridsen H, GiobbieHurder A, et al.: Letrozole therapy alone or in sequence with tamoxifen in women with breast cancer. N Engl J Med 2009;361:766-776. 